\title{
Prospective, randomized, double blind, placebo controlled clinical study to different doses of ketamine for prevention of shivering during spinal anaesthesia
}

\author{
Kanchan R. Rupwate*, Swarda K. Pardeshi, Bharati A. Tendolkar
}

\author{
Department of Anaesthesiology, \\ L.T.M.M.C and L.T.M.G.H, \\ Sion, Mumbai, India \\ Received: 25 July 2016 \\ Accepted: 03 August 2016 \\ *Correspondence to: \\ Dr. Kanchan Rupwate, \\ Email: rupwatekanchan \\ @ rediffmail.com \\ Copyright: () the author(s), \\ publisher and licensee Medip \\ Academy. This is an open- \\ access article distributed under \\ the terms of the Creative \\ Commons Attribution Non- \\ Commercial License, which \\ permits unrestricted non- \\ commercial use, distribution, \\ and reproduction in any \\ medium, provided the original \\ work is properly cited.
}

\begin{abstract}
Background: Ketamine has better effect than other drugs like pethidine, fentanyl, clonidine, tramadol, midazolam in prevention of shivering during anaesthesia and has a role in thermoregulation by different means. The objective of this study was to evaluate the safety, efficacy of Ketamine injection and to compare the different doses $(0.25 \mathrm{mg} / \mathrm{kg}$ and $0.5 \mathrm{mg} / \mathrm{kg})$ of inj. Ketamine in prevention of shivering in operative patients under spinal anaesthesia.

Methods: The present study was a prospective, randomized, double blinded and clinical study conducted in L.T.M.M.C \& L.T.M.G.H, Mumbai, India during January 2012 to September 2013. 120 patients with American Society of Anesthesiologist (ASA) physical status of I or II, between the age of $18-65$ years of either sex and height $150-170 \mathrm{cms}$ were randomly selected and included in the study as per eligibility.

Results: The study suggests that prophylactic administration of injection Ketamine at doses of $0.25 \mathrm{mg} / \mathrm{kg}$ and $0.5 \mathrm{mg} / \mathrm{kg}$ was producing a significant antishivering effect but an incidence of sedation and hallucination was observed in the Ketamine $0.5 \mathrm{mg} / \mathrm{kg}$ receiving group throughout the perioperative period. Conclusions: From this study we can conclude that prophylactic dose of Ketamine $0.25 \mathrm{mg} / \mathrm{kg}$ i.v. has lesser side effects comparison to Ketamine 0.5 $\mathrm{mg} / \mathrm{kg}$ i.v. in prevention of shivering in patients, undergoing surgical procedure under spinal anaesthesia.
\end{abstract}

Keywords: Ketamine, Shivering, Perioperative, Shivering score, Spinal anaesthesia

\section{INTRODUCTION}

Perioperative hypothermia and shivering is among the unwanted, recurrent health issues in surgical patients undergoing both general and regional anaesthesia. Reports say the occurrence of shivering in patients recovering from general anesthesia to be between 5-65\% whereas $40=60 \%$ in patients recovering from regional anesthesia. $^{1-3}$

Although unintended hypothermia is may provide protection against ischemia, there are also several clinical reports which indicate multiple physiological derangements include coagulation abnormalities due to impaired platelet function, wound infection with delayed wound healing due to impaired immunoregulation etc. caused by hypothermia. ${ }^{4}$ Hypothermia during neuraxial anaesthesia develops initially from core to peripheral redistribution of body heat. Redistribution of body heat during spinal or epidural anaesthesia typically decreases core temperature $0.5-1.0^{\circ} \mathrm{C}^{5}$

Shivering is another convoluted response of hypothermia, and is defined as tremor of the face, jaw, head, trunk or extremities lasting longer than 15 seconds. ${ }^{6}$ It causes augmented consumption of oxygen, arterial hypoxia and myocardial ischemia. It also raises intracranial and intraocular pressure. The other effects include increase in 
cardiac output, peripheral resistance, production of carbon dioxide and lactic acidosis. Moreover it also interferes with ECG and oxygen saturation monitoring (pulse oximetry). ${ }^{2,7}$

Various non-pharmacological and pharmacological measures have been studied to control intraoperative shivering which include covering bared body parts with surgical drapes or blankets, airway heating and humidification, warming intravenous fluids and active cutaneous warming with insulator. In addition, a variety of medications are used which include drugs like Injection pethidine, fentanyl, clonidine, tramadol, midazolam.

Recently ketamine has been reported with better results in prevention of shivering during anaesthesia. It is a competitive receptor antagonist of $\mathrm{N}$-methyl-d-aspartic acid (NMDA) has a role in thermoregulation by different means. ${ }^{8}$ Ketamine controls shivering by non-shivering thermogenesis either by the action on the hypothalamus or by the $\beta$-adrenergic effect of Norepinephrine. ${ }^{9}$ It is used as antishivering agent in dose of $0.25-0.75 \mathrm{mg} / \mathrm{kg}$ i.v. ${ }^{3,10}$

Thus, the authors were designed this placebo controlled, randomized, double blinded, prospective study to evaluate the safety, efficacy of inj. Ketamine and to compare the different doses $(0.25 \mathrm{mg} / \mathrm{kg}$ and $0.5 \mathrm{mg} / \mathrm{kg})$ of inj. Ketamine in prevention of shivering in operative patients under spinal anaesthesia. It was also aimed to compare the adverse reactions, if any.

\section{METHODS}

The present study was a prospective, randomized, double blinded and clinical study conducted in L.T.M.M.C \& L.T.M.G.H, Mumbai, India during January 2012 to September 2013. After approval of the institutional medical ethics committee 120 patients with American Society of Anesthesiologist (ASA) physical status of I or II, between the age of 18-65 years of either sex and height $150-170 \mathrm{cms}$ were included in the study. Written informed consent was obtained from all patients to be included in the study. Patient unwilling for consent, with thyroid disorder, severe cardiopulmonary disease, requiring transfusion of blood and blood products, endoscopic urological procedures, pregnancy, BMI $>30 \mathrm{~kg} / \mathrm{m}^{2}$, contraindication for central neuraxial blockade, Coaugulopathy, $\mathrm{H} / \mathrm{O}$ any allergy to local anaesthetic were excluded from the study.

Eligible 120 patients were distributed into 3 groups, each parallel group containing 40 patients each. The patients scheduled to undergo infraumbilical surgical procedures including hernioplasty, appendicectomy, gynaecological procedures, urological procedures and orthopedics procedures lasting upto 150 minutes under spinal anaesthesia were enrolled in the study. After intrathecal injection, $4 \mathrm{ml}$ of one of the study drug was given i.v. bolus. Group K was given Inj Ketamine $0.25 \mathrm{mg} / \mathrm{kg}$ i.v.; group $\mathrm{O}$ was treated with Inj Ketamine $0.5 \mathrm{mg} / \mathrm{kg}$ i.v. and Group P was the placebo or control group.

A complete preoperative assessment was carried out in all patients. Patients selected were randomized in three groups using chit block method allotting equal number of patients in each group. ${ }^{11}$ A syringe with $4 \mathrm{ml}$ of study drug was prepared by an anaesthesiologist, who was not involved in administration of subarchanoid block or recording the outcome. Temperature of operation theatre was checked and maintained at $22-26{ }^{\circ} \mathrm{C}$. Anaesthetic drugs and equipments were kept ready before starting procedure.

After confirmation of starvation status and consent, patient was taken in operation theatre. Monitors like electrocardiogram, pulse-oximeter and NIBP was attached. Core temperature was recorded with the help of nasopharyngeal temperature probe. Patients with baseline temperature $<35{ }^{\circ} \mathrm{C}$ or $>38{ }^{\circ} \mathrm{C}$ were excluded from study. The base line parameters such as heart rate, systolic blood pressure (SBP), diastolic blood pressure (DBP), mean arterial pressure (MAP), $\mathrm{SpO}_{2}$ and core temperature were recorded. An IV line was secured and patient was preloaded with IV fluid (Ringer's Lactate) at the rate of $10 \mathrm{ml} / \mathrm{kg} / \mathrm{hr}$. The preloading IV fluids and the IV infusions, kept at room temperature were used during operation.

Procedure was explained to the patient. Under all aseptic precautions, subarachnoid block was given in sitting position, in L3-L4 space, by midline approach with Quincke's spinal needle no. 25. After confirming free and clear flow of CSF and negative aspiration of blood, Inj. Bupivacaine $0.5 \%$ (Heavy) $3.5 \mathrm{cc}$ was injected. Supine position was given. A standard double layered bed sheet was used to cover the chest and upper limbs of all patients.

Just $5 \mathrm{~min}$ after the intrathecal injection, $4 \mathrm{ml}$ of one of the study drugs was given as IV bolus. The level of sensory block with help of pinprick test was assessed every 5 minutes intervals. The presence of shivering was observed. Patients with inadequate sensory level <T8 fifteen minutes after administration of sub archanoid block or severe pain intraoperatively were excluded from the study.

Parameters including core temperature with the help of nasopharyngeal temperature probe, heart rate, blood pressure, $\mathrm{SpO}_{2}$, electrocardiography monitoring, shivering score, Sedation score were noted and monitored every 15 min till end of surgery and every 30 minutes for 2 hours in postoperative period:

Shivering was graded using a scale similar to that validated by Tsai and Chu. ${ }^{2}$

Grade 0: no shivering; 
Grade 1: piloerection or peripheral vasoconstriction but no visible shivering;

Grade 2: muscular activity in only one muscle group;

Grade 3: muscular activity in more than one muscle group but not generalized;

Grade 4: shivering involving the whole body.

If 15 minutes after spinal anaesthesia and concomitant administration of a prophylactic dose of one of the study drugs, the patients shivered to at least grade 3, shivering was considered significant and prophylaxis as ineffective. Injection Tramadol $1 \mathrm{mg} / \mathrm{kg}$ IV slowly was given as a rescue drug.

The degree of sedation was assessed on 5 point scale where, 1: fully awake and oriented patient, 2: Drowsy, 3: eyes closed, arousable on command, 4: eyes closed, arousable to physical stimuli, 5: eyes closed and patient unarousable to physical stimuli. All patients were given oxygen at rate of $6 \mathrm{ltr} / \mathrm{min}$ with Hadson's mask in intraoperative period and monitored every $15 \mathrm{~min}$ till the end of surgery and every $30 \mathrm{~min}$ till 2 hours.

\section{Statistical data}

Data was expressed as mean \pm standard deviation or median. Demographic data and complications were analyzed using Pearson's chi square test and hemodynamic variables; core temperature, shivering score and sedation score were analyzed using KruskalWallis one way analysis of variance on ranks test. $\mathrm{P}$ values $<0.05$ were considered as significant.

\section{RESULTS}

Prospective, randomized, double blind, placebo controlled clinical study, designed to evaluate and compare the efficacy of ketamine at doses of $(0.25 \mathrm{mg} / \mathrm{kg}$ i.v. $)$ and $(0.5$ $\mathrm{mg} / \mathrm{kg}$ i.v.) for prevention of shivering during spinal anaesthesia. 120 patients of either sex (18-65 yrs) were randomly allocated in 3 groups (Group O: Inj Ketamine $0.5 \mathrm{mg} / \mathrm{kg}$ i.v.; Group K: Inj Ketamine $0.25 \mathrm{mg} / \mathrm{kg}$ i.v. and Group P: placebo or saline group) each parallel groups containing 40 patients each.

Table 1 shows demographic data including age, weight, height and body mass index. Demographic characteristics were compared using Kruskal-Wallis one way analysis of variance on ranks test. All the three groups were comparable, in view of demographic parameters, including age, weight, height and body mass index. Statistically, there was no significant difference.

Sex ratio, sensory level, ASA class are given in Table 2. These characteristics were compared using Pearson chi square test. All the three groups were comparable. Statistically, there was no statistically significant difference.

All the three groups were compared for perioperative core temperature at different time intervals. Baseline core temperatures of all the three groups were comparable. The groups showed highest temperature initially. Difference was statistically significant in all the three groups, at all time intervals except at baseline core temperature.

Table 1: Demographic data of the patients.

\begin{tabular}{|c|c|c|c|c|c|}
\hline Study parameter & & Group 0 & Group K & Group P & p value \\
\hline \multirow{4}{*}{ Age (years) } & Mean & 39.40 & 39.53 & 39.48 & \multirow{4}{*}{0.975} \\
\hline & Std.Dev. & 8.26 & 9.69 & 7.80 & \\
\hline & Median & 39.00 & 39.00 & 38.00 & \\
\hline & IQR & 13.00 & 14.50 & 12.50 & \\
\hline \multirow{4}{*}{ Weight (kgs) } & Mean & 59.58 & 61.08 & 59.70 & \multirow{4}{*}{0.777} \\
\hline & Std.Dev. & 9.01 & 9.29 & 7.94 & \\
\hline & Median & 58.00 & 60.00 & 60.00 & \\
\hline & IQR & 13.50 & 14.50 & 11.50 & \\
\hline \multirow{4}{*}{ Height (cm) } & Mean & 155.18 & 156.15 & 156.60 & \multirow{4}{*}{0.373} \\
\hline & Std.Dev. & 4.86 & 4.87 & 5.29 & \\
\hline & Median & 154 & 154 & 155 & \\
\hline & IQR & 4.50 & 6.00 & 6.50 & \\
\hline \multirow{4}{*}{ BMI $\left(\mathrm{kg} / \mathrm{m}^{2}\right)$} & Mean & 24.58 & 24.85 & 24.21 & \multirow{4}{*}{0.918} \\
\hline & Std.Dev. & 2.62 & 2.54 & 2.06 & \\
\hline & Median & 24.0 & 25.0 & 24.5 & \\
\hline & IQR & 4.85 & 3.75 & 2.95 & \\
\hline
\end{tabular}

$\mathrm{P}<0.05$-significant 
Table 2: Sex, Sensory blockage, ASA class of the patients.

\begin{tabular}{|c|c|c|c|c|c|}
\hline Study Parameters & & Group 0 & Group K & Group P & p value \\
\hline \multirow{2}{*}{$\operatorname{Sex}(M / F)$} & M & $21(52.5 \%)$ & $18(45 \%)$ & $28(70 \%)$ & \multirow{2}{*}{0.068} \\
\hline & $\mathrm{F}$ & $19(47.5 \%)$ & $22(55 \%)$ & $12(30 \%)$ & \\
\hline \multirow{2}{*}{ Sensory level (T6/T7) } & T6 & $28(70 \%)$ & $23(57.5 \%)$ & $32(80 \%)$ & \multirow{2}{*}{0.092} \\
\hline & T7 & $12(30 \%)$ & $17(42.5 \%)$ & $8(20 \%)$ & \\
\hline \multirow{2}{*}{ ASA class (I/II) } & I & $25(62.5 \%)$ & $31(77.5 \%)$ & $28(70 \%)$ & \multirow{2}{*}{0.343} \\
\hline & II & $15(37.5 \%)$ & $9(22.5 \%)$ & $12(30 \%)$ & \\
\hline
\end{tabular}

$\mathrm{P}<0.05$-significant

Table 3: Perioperative core temperature.

\begin{tabular}{|c|c|c|c|c|c|c|}
\hline Study group & Study parameters & Mean & Std.Dev. & Median & IQR & $P$ value \\
\hline Group O & \multirow{3}{*}{ Core temp BL } & 36.70 & 0.46 & 36.80 & 0.75 & \\
\hline Group K & & 36.77 & 0.38 & 37.00 & 0.60 & $0.800^{\mathrm{NS}}$ \\
\hline Group P & & 36.69 & 0.58 & 37.00 & 1.25 & \\
\hline Group O & \multirow{3}{*}{15 mins } & 36.58 & 0.48 & 36.75 & 0.90 & \\
\hline Group K & & 36.56 & 0.35 & 36.60 & 0.40 & 0.006 \\
\hline Group P & & 36.25 & 0.57 & 36.30 & 0.90 & \\
\hline Group O & \multirow{3}{*}{30 mins } & 36.49 & 0.55 & 36.75 & 1.10 & \\
\hline Group K & & 36.42 & 0.43 & 36.55 & 0.45 & 0.000 \\
\hline Group P & & 36.09 & 0.50 & 36.25 & 0.90 & \\
\hline Group O & \multirow{3}{*}{45 mins } & 36.50 & 0.46 & 36.65 & 1.00 & \\
\hline Group K & & 36.40 & 0.36 & 36.55 & 0.55 & 0.040 \\
\hline Group P & & 36.18 & 0.51 & 36.10 & 0.50 & \\
\hline Group O & \multirow{3}{*}{60 mins } & 36.43 & 0.54 & 36.60 & 0.80 & \\
\hline Group K & & 36.55 & 0.32 & 36.55 & 0.35 & 0.006 \\
\hline Group P & & 36.31 & 0.33 & 36.40 & 0.45 & \\
\hline Group O & \multirow{3}{*}{75 mins } & 36.36 & 0.54 & 36.40 & 0.70 & \\
\hline Group K & & 36.59 & 0.24 & 36.60 & 0.40 & 0.001 \\
\hline Group P & & 36.33 & 0.28 & 36.30 & 0.30 & \\
\hline Group O & \multirow{3}{*}{90 mins } & 36.37 & 0.56 & 36.40 & 1.30 & \\
\hline Group K & & 36.71 & 0.19 & 36.80 & 0.30 & 0.039 \\
\hline Group P & & 36.48 & 0.36 & 36.35 & 0.60 & \\
\hline
\end{tabular}

$\mathrm{p}<0.05$-significant; NS-Statistically not significant

All the three groups were compared for pulse rate perioperatively, at different time intervals. Baseline pulse rates of all the three groups were comparable. Highest pulse rate was shown after 15 and 30 minutes. Difference was statistically significant in all the three groups, at alltime intervals except at baseline pulse rate.

Baseline mean arterial pressures of all the three groups were comparable and presented in Table 5. All the three groups were compared for mean arterial pressure perioperatively, at different time intervals. Difference was statistically significant in all the three groups, at alltime intervals except at baseline mean arterial pressure.

Table 6 presents perioperative shivering score and all the three groups were compared at different time intervals. Difference was statistically significant in all the three groups, at all-time intervals. Group $\mathrm{p}$ showed highest score after 30 minutes which gradually decreased with increase of time.

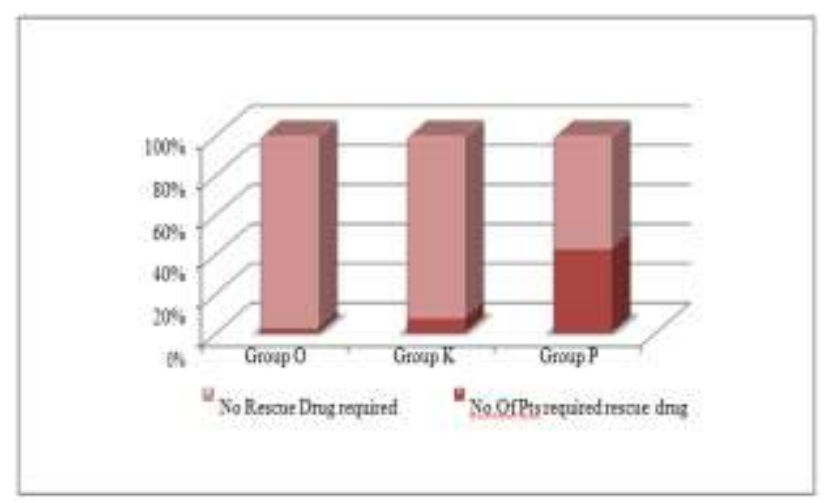

Figure 1: Number of patients required rescue drug. 
Table 4: Perioperative pulse rate.

\begin{tabular}{|ll|lllll|}
\hline $\begin{array}{l}\text { Study } \\
\text { group }\end{array}$ & Study parameters & Mean & Std. Dev. & Median & IQR & P value \\
\hline Group O & PR BL & 69.43 & 6.78 & 68.50 & 9.00 & $0.080^{\text {NS }}$ \\
\hline Group K & & 72.63 & 8.05 & 71.50 & 10.00 & \\
\hline Group P & & 72.88 & 7.89 & 75.00 & 13.50 & \\
\hline Group O & 15 mins & 82.58 & 8.11 & 80.00 & 12.50 & 0.000 \\
\hline Group K & & 81.03 & 8.17 & 85.00 & 13.00 & \\
\hline Group P & & 68.10 & 8.25 & 66.00 & 6.00 & \\
\hline Group O & 30 mins & 80.65 & 4.61 & 82.00 & 8.50 & 0.000 \\
\hline Group K & & 81.13 & 7.78 & 82.00 & 12.00 & \\
\hline Group P & & 68.95 & 9.98 & 68.00 & 5.00 & \\
\hline Group O & 45 mins & 79.68 & 5.54 & 79.00 & 11.00 & 0.000 \\
\hline Group K & & 79.25 & 6.89 & 81.50 & 10.00 & \\
\hline Group P & & 71.13 & 7.45 & 70.00 & 12.00 & \\
\hline Group O & 60 mins & 78.68 & 6.08 & 78.50 & 11.00 & 0.000 \\
\hline Group K & & 79.08 & 6.12 & 80.00 & 10.00 & \\
\hline Group P & & 73.05 & 9.48 & 70.00 & 14.00 & \\
\hline Group O & 75 mins & 76.55 & 6.52 & 76.00 & 10.00 & 0.001 \\
\hline Group K & & 77.41 & 5.31 & 78.00 & 9.00 & \\
\hline Group P & & 71.83 & 9.06 & 69.00 & 13.50 & \\
\hline Group O & 90 mins & 76.28 & 8.09 & 72.00 & 11.00 & 0.001 \\
\hline Group K & & 77.81 & 2.18 & 76.00 & 4.00 & \\
\hline Group P & & 71.69 & 8.69 & 70.00 & 9.50 & \\
\hline
\end{tabular}

$\mathrm{p}<0.05$-significant; NS-Statistically not significant

Table 5: Perioperative mean arterial pressure.

\begin{tabular}{|c|c|c|c|c|c|c|}
\hline Study Group & Study Parameter & Mean & Std.Dev. & Median & IQR & P value \\
\hline Group O & \multirow{3}{*}{ MAP BL } & 96.10 & 8.30 & 96.00 & 10.00 & \\
\hline Group K & & 95.10 & 7.55 & 93.00 & 8.00 & $0.626^{\mathrm{NS}}$ \\
\hline Group P & & 95.18 & 8.56 & 96.00 & 15.00 & \\
\hline Group O & \multirow{3}{*}{15 mins } & 100.90 & 9.21 & 98.00 & 17.00 & \\
\hline Group K & & 94.05 & 8.74 & 95.00 & 10.00 & 0.000 \\
\hline Group P & & 81.38 & 6.79 & 82.00 & 6.50 & \\
\hline Group O & \multirow{3}{*}{30 mins } & 102.40 & 16.69 & 101.00 & 7.00 & \\
\hline Group K & & 93.18 & 8.74 & 98.00 & 11.00 & 0.000 \\
\hline Group P & & 80.15 & 6.30 & 81.00 & 12.00 & \\
\hline Group O & \multirow{3}{*}{45 mins } & 97.75 & 7.41 & 98.50 & 9.00 & \\
\hline Group K & & 91.45 & 8.12 & 93.00 & 6.00 & 0.000 \\
\hline Group P & & 82.25 & 7.66 & 82.00 & 9.00 & \\
\hline Group O & \multirow{3}{*}{60 mins } & 96.05 & 6.35 & 96.00 & 7.00 & \\
\hline Group K & & 91.05 & 6.97 & 93.00 & 5.00 & 0.000 \\
\hline Group P & & 83.00 & 6.84 & 83.00 & 7.50 & \\
\hline Group O & \multirow{3}{*}{75 mins } & 95.40 & 6.50 & 95.00 & 10.00 & \\
\hline Group K & & 91.44 & 6.56 & 93.00 & 11.00 & 0.000 \\
\hline Group P & & 85.13 & 6.00 & 84.50 & 8.00 & \\
\hline Group O & \multirow{3}{*}{90 mins } & 95.56 & 5.33 & 98.50 & 11.00 & \\
\hline Group K & & 85.67 & 3.43 & 84.00 & 8.00 & 0.000 \\
\hline Group P & & 87.07 & 6.34 & 86.00 & 7.50 & \\
\hline
\end{tabular}

$\mathrm{p}<0.05$-significant; NS-Statistically not significant 
Table 6: Perioperative shivering score.

\begin{tabular}{|c|c|c|c|c|c|c|}
\hline Study Gr & Study Parameter & Mean & Std. Dev. & Median & IQR & P value \\
\hline Group O & 15 mins & 0.10 & 0.38 & 0.00 & 0.00 & \\
\hline Group K & & 0.53 & 0.60 & 0.00 & 1.00 & 0.000 \\
\hline Group P & & 1.55 & 0.93 & 2.00 & 1.00 & \\
\hline Group O & 30 mins & 0.28 & 0.72 & 0.00 & 0.00 & \\
\hline Group K & & 0.58 & 0.78 & 0.00 & 1.00 & 0.000 \\
\hline Group P & & 2.13 & 0.72 & 2.00 & 0.00 & \\
\hline Group O & 45 mins & 0.25 & 0.49 & 0.00 & 0.00 & \\
\hline Group K & & 0.78 & 0.83 & 1.00 & 1.00 & 0.000 \\
\hline Group P & & 1.63 & 0.67 & 2.00 & 1.00 & \\
\hline Group O & 60 mins & 0.58 & 0.50 & 1.00 & 1.00 & \\
\hline Group K & & 0.33 & 0.47 & 0.00 & 1.00 & 0.000 \\
\hline Group P & & 1.15 & 0.36 & 1.00 & 0.00 & \\
\hline Group O & 75 mins & 0.55 & 0.50 & 1.00 & 1.00 & \\
\hline Group K & & 0.05 & 0.22 & 0.00 & 0.00 & 0.000 \\
\hline Group P & & 1.10 & 0.50 & 1.00 & 0.00 & \\
\hline Group O & 90 mins & 0.61 & 0.50 & 1.00 & 1.00 & \\
\hline Group K & & 0.05 & 0.21 & 0.00 & 0.00 & 0.000 \\
\hline Group P & & 0.83 & 0.38 & 1.00 & 0.00 & \\
\hline
\end{tabular}

$\mathrm{p}<0.05$-significant

Table 7: Perioperative sedation score.

\begin{tabular}{|c|c|c|c|c|c|c|}
\hline Study group & Study parameters & Mean & Std. Dev. & Median & IQR & P value \\
\hline Group O & \multirow{3}{*}{15 mins } & 2.85 & 0.43 & 3.00 & 0.00 & \\
\hline Group K & & 1.73 & 0.55 & 2.00 & 1.00 & 0.000 \\
\hline Group P & & 1.10 & 0.30 & 1.00 & 0.00 & \\
\hline Group O & \multirow{3}{*}{30 mins } & 2.70 & 0.52 & 3.00 & 1.00 & \\
\hline Group K & & 1.35 & 0.53 & 1.00 & 1.00 & 0.000 \\
\hline Group P & & 1.35 & 0.58 & 1.00 & 1.00 & \\
\hline Group O & \multirow{3}{*}{45 mins } & 2.08 & 0.42 & 2.00 & 0.00 & \\
\hline Group K & & 1.18 & 0.50 & 1.00 & 0.00 & 0.000 \\
\hline Group P & & 1.55 & 0.88 & 1.00 & 1.50 & \\
\hline Group O & \multirow{3}{*}{60 mins } & 1.98 & 0.28 & 2.00 & 0.00 & \\
\hline Group K & & 1.13 & 0.33 & 1.00 & 0.00 & 0.000 \\
\hline Group P & & 1.58 & 0.78 & 1.00 & 1.00 & \\
\hline Group O & \multirow{3}{*}{75 mins } & 1.83 & 0.38 & 2.00 & 0.00 & \\
\hline Group K & & 1.08 & 0.27 & 1.00 & 0.00 & 0.000 \\
\hline Group P & & 1.63 & 0.87 & 1.00 & 1.50 & \\
\hline Group O & \multirow{3}{*}{90 mins } & 1.72 & 0.46 & 2.00 & 1.00 & \\
\hline Group K & & 1.05 & 0.21 & 1.00 & 0.00 & 0.000 \\
\hline Group P & & 1.44 & 0.73 & 1.00 & 1.00 & \\
\hline
\end{tabular}

$\mathrm{p}<0.05$-significant

Figure 1 shows number of patients who required rescue drug (i.e. Tramadol $1 \mathrm{mg} / \mathrm{kg}$ ) when patient shivered to Grade 3 or more than 3. Group P had maximum no. of patients who required Rescue Drug as compared to Group $\mathrm{O}$ and Group K. Difference was statistically significant. $(\mathrm{p}<0.05)$.

All the three groups were compared for Sedation score perioperatively, at different time intervals which was tabulated in Table 7. Difference was statistically significant in all the three groups, at all time intervals.
All the three groups were compared for core temperature postoperatively and presented in Table 8 . The values of the groups after 30 minutes are statistically significant. Postoperative core temperatures, except at $30 \mathrm{mins}$, of all the three groups were comparable.

All the three groups were compared for pulse rate postoperatively, at different time intervals. Difference was statistically significant in all the three groups, at all time intervals. 
Table 8: Postoperative core temperature.

\begin{tabular}{|c|c|c|c|c|c|c|}
\hline Study Gr & Study Parameter & Mean & Std.Dev. & Median & IQR & P value \\
\hline Group O & $\begin{array}{l}\text { PO Core temp } 0 \\
\text { min }\end{array}$ & 36.43 & 0.40 & 36.50 & 0.80 & \\
\hline Group K & & 36.46 & 0.23 & 36.50 & 0.40 & $0.538^{\mathrm{NS}}$ \\
\hline Group P & & 36.48 & 0.37 & 36.50 & 0.40 & \\
\hline Group O & 30 mins & 36.32 & 0.42 & 36.30 & 0.75 & \\
\hline Group K & & 36.35 & 0.21 & 36.40 & 0.20 & 0.001 \\
\hline Group P & & 36.51 & 0.34 & 36.50 & 0.30 & \\
\hline Group O & 60 mins & 36.39 & 0.32 & 36.30 & 0.30 & \\
\hline Group K & & 36.43 & 0.27 & 36.40 & 0.50 & $0.338^{\mathrm{NS}}$ \\
\hline Group P & & 36.45 & 0.35 & 36.50 & 0.60 & \\
\hline Group O & 90 mins & 36.39 & 0.27 & 36.30 & 0.30 & \\
\hline Group K & & 36.46 & 0.34 & 36.60 & 0.70 & $0.377^{\mathrm{NS}}$ \\
\hline Group P & & 36.47 & 0.44 & 36.50 & 1.00 & \\
\hline Group O & $\begin{array}{l}\text { PO Core temp } 120 \\
\text { mins }\end{array}$ & 36.38 & 0.33 & 36.30 & 0.70 & \\
\hline Group K & & 36.41 & 0.35 & 36.40 & 0.45 & $0.914^{\mathrm{NS}}$ \\
\hline Group P & & 36.42 & 0.42 & 36.20 & 0.90 & \\
\hline
\end{tabular}

$\mathrm{p}<0.05$-significant; NS-Statistically not significant

Table 9: Postoperative pulse rate.

\begin{tabular}{|c|c|c|c|c|c|c|}
\hline Study Group & Study Parameter & Mean & Std.Dev. & Median & IQR & P value \\
\hline Group O & 0 mins & 75.55 & 7.31 & 72.00 & 12.00 & \\
\hline Group K & & 73.88 & 5.81 & 70.00 & 10.00 & 0.041 \\
\hline Group P & & 70.60 & 8.18 & 72.00 & 18.00 & \\
\hline Group O & 30 mins & 76.30 & 7.04 & 73.50 & 7.50 & \\
\hline Group K & & 75.20 & 4.78 & 74.00 & 4.50 & 0.039 \\
\hline Group P & & 71.55 & 7.18 & 74.00 & 10.00 & \\
\hline Group O & 60 mins & 74.70 & 6.70 & 72.00 & 11.00 & \\
\hline Group K & & 74.93 & 2.89 & 75.00 & 5.00 & 0.000 \\
\hline Group P & & 70.88 & 6.70 & 70.00 & 10.50 & \\
\hline Group O & 90 mins & 74.00 & 7.16 & 71.00 & 9.00 & \\
\hline Group K & & 75.08 & 3.74 & 76.00 & 6.50 & 0.000 \\
\hline Group P & & 69.78 & 6.29 & 70.00 & 5.00 & \\
\hline Group O & 120 mins & 73.30 & 7.74 & 70.00 & 11.00 & \\
\hline Group K & & 74.45 & 5.47 & 71.00 & 10.00 & 0.006 \\
\hline Group P & & 70.05 & 6.77 & 70.00 & 5.00 & \\
\hline
\end{tabular}

$\mathrm{p}<0.05$-significant

Table 11 showed shivering score postoperatively, at different time intervals. Highest score was found in case of Group $\mathrm{P}$ in 0 minutes followed by 30 minutes. Difference was statistically significant in all the three groups, at all time intervals.

Sedation score postoperatively is given in Table 12 at different time intervals. Group $\mathrm{O}$ with 0 minute followed by 30 minutes showed higher results in comparison to other groups. Difference was statistically significant in all the three groups, at all time intervals except at 120 mins.

Table 13 shows perioperative complications among all the three groups. In Group O, 20\% patients had hallucinations and that of Group $\mathrm{K}$ only $2.5 \%$ patients had hallucinations. Difference was statistically significant. Nausea and vomiting was seen only in Group $\mathrm{P}$, incidence being $10 \%$.There was no incidence of bradycardia, hypotension and hypertension noted in any group.

All the three groups were compared for Mean Arterial pressure postoperatively, at different time intervals and presented in Table 10. Group o showed highest value after 30 minutes. Difference was statistically significant in all the three groups, at all time intervals. 
Table 10: Postoperative mean arterial pressure.

\begin{tabular}{|c|c|c|c|c|c|c|}
\hline Study group & Study Parameters & Mean & Std.Dev. & Median & IQR & $P$ value \\
\hline Group O & \multirow{3}{*}{ PO MAP 0 mins } & 95.80 & 7.12 & 97.00 & 8.00 & \\
\hline Group K & & 90.40 & 5.91 & 93.00 & 10.00 & \multirow{2}{*}{0.000} \\
\hline Group P & & 85.13 & 7.44 & 83.00 & 5.50 & \\
\hline Group O & \multirow{3}{*}{$30 \mathrm{mins}$} & 96.28 & 9.34 & 96.00 & 14.50 & \\
\hline Group K & & 90.65 & 6.70 & 90.50 & 9.50 & \multirow{2}{*}{0.000} \\
\hline Group P & & 85.95 & 7.58 & 82.00 & 9.00 & \\
\hline Group O & \multirow{3}{*}{60 mins } & 95.18 & 9.16 & 99.00 & 16.00 & \\
\hline Group K & & 91.68 & 8.85 & 95.50 & 16.00 & \multirow{2}{*}{0.000} \\
\hline Group P & & 86.73 & 7.75 & 83.50 & 11.00 & \\
\hline Group O & \multirow{3}{*}{90 mins } & 91.93 & 5.83 & 93.00 & 8.00 & \multirow{3}{*}{0.000} \\
\hline Group K & & 91.50 & 6.76 & 93.00 & 7.00 & \\
\hline Group P & & 86.60 & 5.90 & 85.00 & 11.50 & \\
\hline Group O & \multirow{3}{*}{ PO MAP 120 mins } & 93.53 & 8.81 & 96.50 & 20.00 & \\
\hline Group K & & 91.78 & 6.47 & 93.00 & 5.00 & \multirow{2}{*}{0.003} \\
\hline Group P & & 87.38 & 6.04 & 84.00 & 9.50 & \\
\hline
\end{tabular}

$\mathrm{p}<0.05$-significant

Table 11: Postoperative shivering score.

\begin{tabular}{|c|c|c|c|c|c|c|}
\hline Study group & Study parameters & Mean & Std.Dev. & Median & IQR & P value \\
\hline Group O & \multirow{3}{*}{ PO Shivering Sc 0 mins } & 0.48 & 0.51 & 0.00 & 1.00 & \\
\hline Group K & & 0.93 & 0.57 & 1.00 & 0.00 & \multirow{2}{*}{0.000} \\
\hline Group P & & 1.60 & 0.63 & 2.00 & 1.00 & \\
\hline Group O & \multirow{3}{*}{30 mins } & 0.28 & 0.51 & 0.00 & 0.50 & \\
\hline Group K & & 0.65 & 0.62 & 1.00 & 1.00 & \multirow{2}{*}{0.000} \\
\hline Group P & & 1.33 & 0.62 & 1.00 & 1.00 & \\
\hline Group O & \multirow{3}{*}{60 mins } & 0.28 & 0.64 & 0.00 & 0.00 & \\
\hline Group K & & 0.00 & 0.00 & 0.00 & 0.00 & \multirow{2}{*}{0.000} \\
\hline Group P & & 0.88 & 0.40 & 1.00 & 0.00 & \\
\hline Group O & \multirow{3}{*}{90 mins } & 0.35 & 0.70 & 0.00 & 0.00 & \multirow{3}{*}{0.000} \\
\hline Group K & & 0.03 & 0.16 & 0.00 & 0.00 & \\
\hline Group P & & 0.48 & 0.51 & 0.00 & 1.00 & \\
\hline Group O & \multirow{3}{*}{ PO Shivering Sc 120 mins } & 0.35 & 0.70 & 0.00 & 0.00 & \\
\hline Group K & & 0.03 & 0.16 & 0.00 & 0.00 & \multirow{2}{*}{0.006} \\
\hline Group P & & 0.30 & 0.46 & 0.00 & 1.00 & \\
\hline
\end{tabular}

(p<0.05-significant)

Table 12: Postoperative sedation score.

\begin{tabular}{|c|c|c|c|c|c|c|}
\hline Study group & Study parameters & Mean & Std.Dev. & Median & IQR & P value \\
\hline Group O & \multirow{3}{*}{ PO Sedation sc 0 mins } & 2.18 & 0.50 & 2.00 & 0.00 & \\
\hline Group K & & 1.28 & 0.45 & 1.00 & 1.00 & \multirow{2}{*}{0.000} \\
\hline Group P & & 1.53 & 0.72 & 1.00 & 1.00 & \\
\hline Group O & \multirow{3}{*}{30 mins } & 1.98 & 0.28 & 2.00 & 0.00 & \\
\hline Group K & & 1.13 & 0.33 & 1.00 & 0.00 & \multirow{2}{*}{0.000} \\
\hline Group P & & 1.48 & 0.72 & 1.00 & 1.00 & \\
\hline Group O & \multirow{3}{*}{60 mins } & 1.78 & 0.42 & 2.00 & 0.00 & \\
\hline Group K & & 1.05 & 0.22 & 1.00 & 0.00 & \multirow{2}{*}{0.000} \\
\hline Group P & & 1.23 & 0.42 & 1.00 & 0.00 & \\
\hline Group O & \multirow{3}{*}{90 mins } & 1.25 & 0.44 & 1.00 & 0.50 & \\
\hline Group K & & 1.03 & 0.16 & 1.00 & 0.00 & \multirow{2}{*}{0.013} \\
\hline Group P & & 1.13 & 0.33 & 1.00 & 0.00 & \\
\hline Group O & \multirow{3}{*}{ PO Sedation sc 120 mins } & 1.00 & 0.00 & 1.00 & 0.00 & \\
\hline Group K & & 1.00 & 0.00 & 1.00 & 0.00 & \multirow{2}{*}{1.000} \\
\hline Group P & & 1.00 & 0.00 & 1.00 & 0.00 & \\
\hline
\end{tabular}

( $\mathrm{p}<0.05$-significant $)$ 
Table 13: Perioperative complications.

\begin{tabular}{|lllll|}
\hline Complications & Group O (n=40) & Group K (n=40) & Group P (n=40) & P value \\
\hline Nausea and vomiting & $0 / 40(0 \%)$ & $0 / 40(0 \%)$ & $4 / 40(10 \%)$ & 0.016 \\
\hline Hallucinations & $8 / 40(20 \%)$ & $1 / 40(2.5 \%)$ & $0 / 40(0 \%)$ & 0.001 \\
\hline Bradycardia & $0 / 40(0 \%)$ & $0 / 40(0 \%)$ & $0 / 40(0 \%)$ & NS \\
\hline Hypotension/Hypertension & $0 / 40(0 \%)$ & $0 / 40(0 \%)$ & $0 / 40(0 \%)$ & NS \\
\hline
\end{tabular}

Data was analysed by using Pearson chi square test. NS-Statistically not significant.

\section{DISCUSSION}

Shivering can be distressing to the patient and has been cited as one of the primary causes of discomfort during the perioperative period. The most significant physiological consequence is an increased oxygen demand to six times that may lead to hypoxia and death. $^{3,12,13}$ Mild hypothermia is accompanied with compromised immunity, delayed wound healing, coagulopathy, increased risk in allergenic blood transfusions, delayed post anaesthetic recovery, prolonged hospitalistion, discomfort, and morbid myocardial outcomes secondary to sympathetic nervous system stimulation. $^{14}$ It is thus imperative that monitoring temperature in patients undergoing general anaesthesia longer than $30 \mathrm{~min}$ and major operations under neuraxial anaesthesia. $^{14}$

Various pharmacological agents have been evaluated for their efficacy in preventing and treating shivering, however, a "gold standard" drug treatment has not been defined. ${ }^{12}$ Previously several number of pharmacological agents are used in preventing and treating shivering but these drugs had a wide-range of unpredictable side effects, including respiratory depression, hypotension, sedation, itching, nausea and vomiting. ${ }^{7}$ Recently ketamine is proved as an good drug in prevent shivering during anaesthesia without causing cardiovascular or respiratory depression. ${ }^{15}$ It controls shivering by nonshivering thermogenesis either by the action on the hypothalamus or by the $\beta$-adrenergic effect of Norepinephrine by decreasing core to peripheral redistribution of heat. ${ }^{16}$

Generally the dose of ketamine for induction and maintenance of general anesthesia is $1-2 \mathrm{mg} / \mathrm{kg}$ but that dose is associated with deleterious side effects. Previous studies proved that ketamine at a doses of $0.25-0.75$ $\mathrm{mg} / \mathrm{kg}$ was considered as an effective antishivering agent during perioperative period with reduced side effects. 16 Hence the present study is aimed to compare the efficacy between two doses of ketamine $0.25 \mathrm{mg} / \mathrm{kg}$ and $0.5 \mathrm{mg} / \mathrm{kg}$ (i.v. bolus) for prevention of shivering during spinal anaesthesia in peri-operative period.

In the present study fall in core temperature was observed in all the groups, as compared to baseline values, but it is less in patients receiving Inj Ketamine. These observations are in accordance with the previous studies done by Kose et al and Sagir et al. ${ }^{17,18}$

The efficacy of ketamine as an antishivering agent was also proved in the studies conducted by Shakya et al. ${ }^{2} \mathrm{He}$ found that fall in temperature was more significant in saline and ondansetron group than in ketamine $(0.25 \mathrm{mg} / \mathrm{kg})$ group at all time intervals. In the study done by Kinoshita et al proved that low-dose ketamine administration may confer thermoprotection during spinal anesthesia sedated by propofol. ${ }^{19}$ This relative preservation of core temperature in the ketamine groups may be attributed to the sympathetic stimulation and vasoconstrictive effects of the drug. ${ }^{7,16}$

In our study perioperative median shivering score was higher in control group and they are required with rescue drug as compared to patients receiving ketamine. These results are similar to the studies carried out by Shakya et $\mathrm{al}^{2}$ In our study, we found that, the incidence of shivering in the control group is $(42.5 \%)$ which is lower (as compared to $55 \%)$; and in the ketamine $(0.5 \mathrm{mg} / \mathrm{kg})$ group it was $2.5 \%$ which is higher to $0 \%$ as in the comparative study conducted by Sagir et al. He compared the efficacy of Ketamine $(0.5 \mathrm{mg} / \mathrm{kg})$, Ketamine $(0.25 \mathrm{mg} / \mathrm{kg})$ plus Granisetron $(1.5 \mathrm{mg})$, Granisetron $(3 \mathrm{mg})$ and saline in patients undergoing urological surgeries In our study it is also found that the Inj Ketamine $0.5 \mathrm{mg} / \mathrm{kg}$ group had lower incidence of shivering (2.5\%) as compared to $18 \%$ in the studies done by Ahmed A. et al. ${ }^{20}$

From the results of our study, we found that incidence of significant shivering in control group was $42.5 \%$ and in Inj Ketamine $0.5 \mathrm{mg} / \mathrm{kg}$ group is $2.5 \%$ that was comparably lower than the study conducted by Wason et al proving the efficacy of ketamine as prophylactic agent during perioperative period. ${ }^{21}$

In a study conducted by Mirza Koeshardiandi et al concluded that ketamine $0.25 \mathrm{mg} / \mathrm{kg}$ i.v. is effective in lowering shivering after spinal anaesthesia in cesarean section either before or after the baby is born, with same effectiveness as pethidine $0.5 \mathrm{mg} / \mathrm{kg}$ and the same outcome were also supported by Dal and his colleagues in a double blind study using normal saline, $0.5 \mathrm{mg} / \mathrm{kg}$ Ketamine and $20 \mathrm{mg}$ Pethidine prophylactically in preventing post operative shivering in patients who underwent general anaesthesia. ${ }^{22,7}$ Results clearly showed 
that prophylactic dose $0.5 \mathrm{mg} / \mathrm{kg}$ of Ketamine and $20 \mathrm{mg}$ of Pethidine were significantly effective and equal in controlling postoperative shivering than the placebo without side effects.

In this study perioperative pulse rate, mean arterial blood pressure was observed to be higher in patients receiving Ketamine as compared to placebo group but did not observed any incidence of tachycardia, bradycardia, hypotension or hypertension. These results are similar with the previous studies of Wason et al. ${ }^{21}$ This relative preservation of hemodynamics in the ketamine groups may be attributed to the sympathetic stimulation and vasoconstrictive effects of the drug.

Perioperative median sedation score were observed more with Ketamine $0.5 \mathrm{mg} / \mathrm{kg}$ i.v. along with a common side effect of hallucination compared to $0.25 \mathrm{mg} / \mathrm{kg}$ received group. These effects are significantly similar to the previous studies of Kose et al, Safavi et al and Abdelrahman et al. ${ }^{10,8,23}$ But other side effects like nystagmus, feeling like walking in space, delirium was not observed in patients after receiving Ketamine 0.75 $\mathrm{mg} / \mathrm{kg}$ which was observed in the previous study done by Kose et al. ${ }^{24}$

The findings of our study suggest that prophylactic administration of injection Ketamine at doses of 0.25 $\mathrm{mg} / \mathrm{kg}$ and $0.5 \mathrm{mg} / \mathrm{kg}$ was producing a significant antishivering effect but an incidence of sedation and hallucination was observed in the Ketamine $0.5 \mathrm{mg} / \mathrm{kg}$ receiving group throughout the perioperative period. In conclusion, prophylactic dose of Ketamine $0.25 \mathrm{mg} / \mathrm{kg}$ i.v. is preferred over ketamine $0.5 \mathrm{mg} / \mathrm{kg}$ i.v. for prevention of shivering in patients, undergoing surgical procedure under spinal anaesthesia, with lesser side effects.

Funding: No funding resources Conflict of interest: None declared

Ethical approval: Approved by institutional ethics committee

\section{REFERENCES}

1. Gangopadhyay S, Gupta K, Acharjee S, Nayak S, Dawn S, Piplai G. Ketamine, Tramadol and Pethidine in Prophylaxis of Shivering During Spinal Anaesthesia. J Anaesth Clin Pharmacol. 2010;26(1):59-63.

2. Shakya B, Chaturvedi A, Sah BP. Prophylactic low dose ketamine and ondansetron for prevention of shivering during spinal anaesthesia. J Anaesthesiol Clin Pharmacol. 2010;26(4):465-9.

3. Norouzi M, Doroodian MR, Salajegheh S. Optimum dose of ketamine for prevention of postanaesthetic shivering; a randomized double-blind placebocontrolled clinical trial. Acta Anaesthesiol Belg. 2011;62(1):33-6.
4. Bhattacharya P, Bhattacharya L, Jain R, Aggarwal R. Post Anaesthesia shivering (PAS): A Review. Indian J Anaesth. 2003;47(2):88-93.

5. Crowley LJ, Buggy DJ. Shivering and neuraxial anaesthesia. Regional Anaesthesia Pain Med. 2008;33(3):241-52.

6. Kose EA, Honca M, Dal D, Akinci SB, Aypar U. Prophylactic ketamine to prevent shivering in parturients undergoing Cesarean delivery during spinal anesthesia. J Clin Anesthesia. 2013;25(4):275-80.

7. Dal D, Kose A, Honca M, Akinci B, Basgul E, Aypar U. Efficacy of prophylactic ketamine in preventing postoperative shivering. $\mathrm{Br} \mathrm{J}$ Anaesth. 2005;95:189-92.

8. Honarmand A, Safavi MR. Comparison of prophylactic use of midazolam, ketamine and ketamine plus midazolam for prevention of shivering during regional anaesthesia: a randomized double blind placebo controlled trial. Br J Anaesth. 2008;101(4):557-62.

9. Sharma DR, Thakur JR. Ketamine and shivering. Anaesthesia. 1990;45:252-3.

10. Kose EA, Honca M, Dal D, Akinci SB, Aypar U. Prophylactic ketamine to prevent shivering in parturients undergoing Cesarean delivery during spinal anesthesia. J Clin Anesth. 2013;25(4):275-80.

11. Tuljapure S, Kotambkar V. A randomised controlled study of tracheal extubation response following nitroglycerine (NTG) sublingual spray in normotensive and hypertensive patients. Indian $\mathbf{J}$ Basic Appl Med Res. 2015;4(3):45-54.

12. Kranke P, Eberhart LH, Roewer N, Tramèr MR. Pharmacological treatment of postoperative shivering: a quantitative systematic review of randomized controlled trials. Anesth Analg. 2002;94(2):453.

13. Alfonsi P. Postanaesthetic shivering: epidemiology, pathophysiology, and approaches to prevention and management. Drugs. 2001;61(15):2193-205.

14. Sessler DI. Temperature Monitoring and Perioperative Thermoregulation. Anaesthesiology. 2008;109(2):318-38.

15. Reves JG, Glass P, Lubarsky DA, McEvoy MD, Martinez-Ruiz R. Intravenous anesthetics. In Miller RD, Eriksson LI, Fleisher LA, Wiener-kronish JP, Young WL (editors). Miller's Anaesthesia, 7th Edition. Philadelphia, Elsevier Churchill Livingstone; 2010: 742-747.

16. Sharma DR, Thakur JR. Ketamine and shivering. Anaesthesia. 1990;45:252-3.

17. Kose EA, Honca M, Dal D, Akinci SB, Aypar U. Prophylactic ketamine to prevent shivering in parturients undergoing Cesarean delivery during spinal anesthesia. J Clin Anesth. 201;25(4):275-80.

18. Sagir O, Gulhas N, Toprak H, Yucel A, Begec Z, Ersoy O. Control of shivering during regional anesthesia: prophylactic Ketamine and Granisteron. Acta Anaesthesiol Scand. 2007;51(1):44-9. 
19. Kinoshita T, Suzuki M, Shimada Y, Ogawa R. Effect of Low-Dose Ketamine on Redistribution Hypothermia during Spinal Anesthesia Sedated by Propofol. J Nippon Med Sch. 2004;71(2):92-8.

20. Ahmed A, Aslam M. Prevention of Shivering during lower segment cesarean section; Comparison of prophylactic use of ketamine and ketamine plus midazolam during spinal anaesthesia. Professional Med J. 2013;20(3):409-15.

21. Wason R, Jain N, Gupta P, Gogia AR. Randomized double-blind comparison of prophylactic ketamine, clonidine and tramadol for the control of shivering under neuraxial anaesthesia. Indian $\mathbf{J}$ Anaesth. 2012;56(4):370-5.
22. Koeshardiandi M, Rehatta N. Effectiveness Dose Ketamine $0.25 \mathrm{mg} / \mathrm{kg}$ i.v. for Shivering as Therapy During Spinal Anesthesia on surgery of cesarean section. Journal of Emergency. 2011;1(1):45-53.

23. Abdelrahman R. Prevention of shivering during Regional anaesthesia: Comparison of Midazolam, Midazolam plus Ketamine, Tramadol, Tramadol plus Ketamine. Life Sci J. 2012;9(2):132-9.

24. Kose EA, Dal D, Akinci SB, Saricaoglu F, Aypar U. The efficacy of ketamine for the treatment of postoperative shivering. Anesth Analg. 2008;106(1):120-2.

Cite this article as: Rupwate KR, Pardeshi SK, Tendolkar BA. Prospective, randomized, double blind, placebo controlled clinical study to different doses of ketamine for prevention of shivering during spinal anaesthesia. Int J Basic Clin Pharmacol 2016;5:1866-76. 